\title{
Understanding the impact of pulmonary arterial hypertension on patients' and carers' lives
}

\author{
Loïc Guillevin', lain Armstrong ${ }^{2}$, Rino Aldrighetti ${ }^{3}$, Luke S. Howard4, \\ Henrik Ryftenius ${ }^{5}$, Aryeh Fischer ${ }^{6}$, Sandra Lombardi ${ }^{7}$, Sean Studer ${ }^{8}$ \\ and Pisana Ferrari ${ }^{9}$
}

\begin{abstract}
Affiliations: 'Service de Médecine Interne, Hôpital Cochin, Paris, France. ${ }^{2}$ Pulmonary Hypertension Association UK, Rotherham, and ${ }^{3}$ National Pulmonary Hypertension Service, Hammersmith Hospital, Imperial College London, London, UK. ${ }^{3}$ Pulmonary Hypertension Association US, Silver Spring, MD, ${ }^{6}$ Division of Rheumatology, Dept of Medicine, National Jewish Health, Denver, C0, ${ }^{7}$ UCSD Medical Center, San Diego, CA, and ${ }^{8}$ Woodhull Medical Center, New York University, New York, NY, USA. ${ }^{5}$ Lung Allergy Clinic, Karolinska University Hospital, Stockholm, Sweden. ${ }^{9}$ Pulmonary Hypertension Association Europe, Vienna, Austria.
\end{abstract}

Correspondence: L. Guillevin, Service de Médecine Interne, Hôpital Cochin, 27 rue du Faubourg Saint-Jacques, F-75014 Paris, France. E-mail: loic.guillevindacch.ap-hop-paris.fr

ABSTRACT Pulmonary arterial hypertension (PAH) is a rare, debilitating and rapidly progressive disease. Although there have been important medical advances in PAH management, the search for a cure continues. Despite an increased understanding of the disease, data on the wider effect of PAH on patients and carers, beyond the clinical symptoms, are still limited. In order to explore this, a large-scale international survey investigated four key areas affected by PAH (physical and practical, emotional, social, and information needs) and provides new insight into patients' and carers' experiences of living with the disease. The results from the survey highlight not only the limited ability of patients to carry out everyday tasks, but also the financial impact and social isolation experienced by both patients and carers. The study confirmed that a decline in a patient's World Health Organization functional class, which indicates an increase in clinical severity of the disease, is associated with greater limitations. Results from the survey demonstrate the need for multidisciplinary PAH management and a comprehensive standard of care to assess and improve all aspects of well-being for both patients and carers. In addition, they underline the need for updated PAH guidelines that address these needs.

@ERSpublications

PAH has a profound impact on numerous aspects of patients' and carers' lives http://ow.ly/pWWYf

This article has supplementary material available from err.ersjournals.com

Full survey results, along with a call to action, are available at www.phaeurope.org/projects-activities/pah-patient-andcarer-survey

Received: Aug 272013 | Accepted after revision: Oct 162013

Support statement: All authors are members of the International PAH Patient And Carer Survey Steering Committee and have received honoraria and travel expenses from Actelion that were paid either to them or to their institution.

Conflict of interest: Disclosures can be found alongside the online version of this article at err.ersjournals.com

Provenance: Publication of this peer-reviewed article was supported by Actelion Pharmaceuticals Ltd, Switzerland (principal sponsor, European Respiratory Review issue 130).

Copyright OERS 2013. ERR articles are open access and distributed under the terms of the Creative Commons Attribution Non-Commercial Licence 3.0. 


\section{Introduction}

Pulmonary arterial hypertension (PAH) is a rare condition characterised by increased pulmonary vascular resistance and arterial pressure ultimately leading to right heart failure and death [1]. PAH symptoms include dyspnoea, fatigue, chest pain, syncope and peripheral oedema [2]. Although significant advances have been made regarding the understanding of the disease and its treatment, it remains a devastating, progressively debilitating disease [1, 3-6].

Research into the impact that PAH has on the lives of patients beyond the clinical symptoms of the disease is limited, and there is virtually no information regarding the impact that PAH has on carers. Measurements of health-related quality of life (QoL) in PAH patients have been reported in a number of studies, and many of these have shown severe impairments in several or all dimensions [7]. However, the majority of these studies used instruments not specifically developed for $\mathrm{PAH}$, and standard generic QoL measures or tools specific to lung or heart disease may not properly reflect the clinical status of PAH $[7,8]$.

A high prevalence of depression and anxiety in PAH patients has been described in several studies. Reported rates were between $20 \%$ and $40 \%$ for anxiety [9-11] and between $21 \%$ and $55 \%$ for depression $[9,11-14$ ], with the exception of one study that reported a lower rate of $8 \%$ [10]. Moreover, neuropsychological tests identified cognitive sequelae in $59 \%$ of $\mathrm{PAH}$ patients, most frequently impairments in motor function $(57 \%)$ and memory $(41 \%)$ [11].

Data on other important aspects regarding the impact of PAH on patients' lives are scarce. It is known that the proportion of $\mathrm{PAH}$ patients unable to work due to their $\mathrm{PAH}$ is considerable; reported figures range between 45\% [9] and 71\% [15]. Therefore, the socioeconomic impact of PAH is likely to merit further exploration [7]. Concerns regarding intimacy is another issue frequently reported by patients and partners, but no research has been carried out in this area [7].

Qualitative interviews have recently been used to investigate patient's experiences of living with PAH, and overarching themes have emerged. These were "coping with uncertainty" and "moving on with life" in one study [16], and "holding back" and "re-defining life" in a second study [17]. Qualitative interviews conducted in view of the design of a pulmonary hypertension ( $\mathrm{PH}$ )-specific tool to measure QoL identified the following PH-specific issues: socialising/interaction with others; being understood, accepted and valued; self-esteem; independence (financial and physical); and security [18].

This position statement has been developed following an International PAH Patient and Carer Survey that investigated the impact of PAH on patients and carers, beyond the clinical symptoms of the disease. This is the first large-scale survey to not only explore the perspective of PAH patients, but also that of carers. The purpose of this position paper is to provide an overview of the survey results and to consider the implications of these findings on the future management of PAH for both patients and carers, which will involve healthcare professionals (HCPs) and other specialists.

In order to address this, an international, large-scale, self-reported survey was conducted, using market research methodologies. Four key areas that are affected by PAH were explored: the physical and practical impact; the emotional impact; the social impact; and information needs and provisions. The aims of the survey were to improve the understanding of patients' and carers' experiences of living with PAH, to inform the scientific community, and to gather evidence to illustrate the need for a more comprehensive standard of PAH care in the future.

A multidisciplinary steering committee of PAH specialist physicians and nurses, as well as patient group representatives, led the development of the survey content and the analysis of results to ensure medical and practical relevance. The survey was conducted in five European countries, providing an international perspective, and was composed of two phases: a qualitative survey based on one-to-one interviews with patients and carers to identify themes to explore further; and a quantitative survey using opinion-based questionnaires to evaluate the impact of $\mathrm{PAH}$ beyond the clinical symptoms. Patients and carers were approached by national patient organisations and by HCPs managing PAH patients. UK versions of the questionnaires are available as supplementary material.

\section{Key findings from the international survey}

A total of 455 respondents participated in the survey, which included $326 \mathrm{PAH}$ patients and 129 carers. Among the carers, 55\% were the spouse or partner of the PAH patient. $21 \%$ and $18 \%$ cared for a child or a parent, respectively, and $5 \%$ and $1 \%$ were other family members or friends of the PAH patient, respectively. As the evaluation of the survey results was performed anonymously, it was not possible to determine whether any of the carers cared for a patient who also responded to the survey. 


\begin{tabular}{lcc} 
& Patients & Carers \\
\hline Subjects $n$ & 326 & 129 \\
Mean age years & 52 & 52 \\
Females \% & 74 & 56 \\
Country & & $29(22)$ \\
France & $70(21)$ & $11(9)$ \\
Germany & $97(30)$ & $50(39)$ \\
Italy & $81(25)$ & $31(24)$ \\
Spain & $45(14)$ & $8(6)$ \\
UK & $33(10)$ & $70(54)$ \\
Type of PAH & & $5(4)$ \\
Idiopathic PAH & $160(49)$ & $13(10)$ \\
Family history of PAH & $16(5)$ & $18(14)$ \\
PAH associated with CTD & $42(13)$ & $15(12)$ \\
PAH associated with CHD & $39(12)$ & $8(6)$ \\
PAH associated with HIV & $7(2)$ & $42(13)$ \\
PAH associated with another disease & $20(6)$ & $40(3)$ \\
Not sure & & $55(43)$ \\
WHO/NYHA functional class & $16(5)$ & $30(23)$ \\
I & $163(50)$ & $111(34)$ \\
II & $36(11)$ &
\end{tabular}

Data are presented as $\mathrm{n}(\%)$, unless otherwise stated. PAH: pulmonary arterial hypertension; CTD: connective tissue disease; CHD: congenital heart disease; WHO: World Health Organization; NYHA: New York Heart Association.

Demographic characteristics of the respondents are summarised in table 1 . The mean age was 52 years both for patients and carers; $74 \%$ of patients and $56 \%$ of carers were female. Idiopathic PAH was the most common aetiology, and most patients were in World Health Organization functional class II or III. The key findings of the survey are described below.

\section{PAH has a significant physical and practical impact on the daily lives of patients and carers}

The physical and practical impact of PAH can vary greatly on a daily basis, and performing everyday tasks can be challenging. Patients reported that their physical activities were severely restricted due to PAH, with $56 \%$ describing how PAH has a very significant impact on their daily lives. The key areas of life affected by $\mathrm{PAH}$ were physical activities, employment/work, travel and social opportunities, dealing with domestic work/household chores, and relationship issues. As expected, a decline in patients' functional class was associated with a greater impact on many aspects of daily life for both patients and carers, with a high proportion of patients with severe disease (functional class III and IV) and their carers reporting a very significant impact on their lives. Caring for a patient with PAH can be physically draining and this is reflected by $57 \%$ of carers feeling that $\mathrm{PAH}$ has a profound effect on day-to-day living, often resulting in exhaustion $(43 \%)$, due to extra tasks and responsibilities.

\section{PAH is associated with a significant financial burden}

The issue that patients felt most concerned or frustrated about was the impact of PAH on their employment. Most patients (85\%) reported that their work or employment had been affected by PAH. The ability of the carers to work was also impacted, with 29\% of carers feeling that their work had been affected by caring for someone with PAH. Not surprisingly, this reduction in ability to work due to PAH had major consequences on overall household incomes, with both patients (73\%) and carers (35\%) reporting a major impact on finances (fig. 1), leaving many families with below average incomes.

\section{PAH has a considerable impact on intimacy and relationships}

Many patients and carers noted a loss of intimacy and diminished sexual relationships due to PAH, which declined further as functional class worsened. Almost all patients reported that their sex lives were severely affected, mainly due to low self-esteem (30\%) and being unable to physically exert themselves (23\%). Nearly three-quarters $(72 \%)$ of carers who were partners of $\mathrm{PAH}$ patients reported a decrease in sexual 


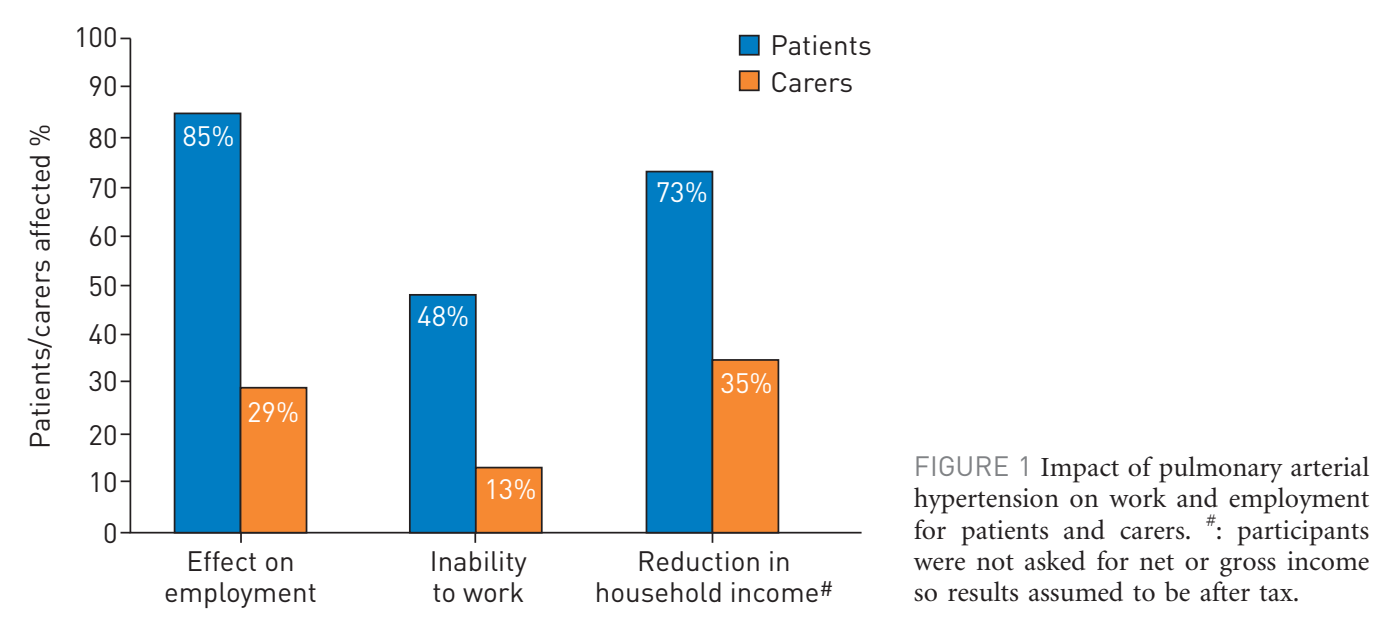

relationships, mainly due to their partner's lack of interest since developing PAH and their own fear of making the patient more ill. As a consequence, one in five carers reported feeling less close to their spouse $(23 \%)$ and feeling that their spouse saw them more as a carer than as a lover (18\%). The impact on sexual relationships increased with a decline in functional class, with virtually all carers affected (93\%) when caring for PAH patients in functional class IV, and $66 \%$ of functional class IV patients experiencing a loss of libido often or very often.

\section{PAH has a heavy burden on emotional and social well-being}

Patients and carers frequently reported that PAH has a heavy burden on their social and emotional wellbeing. Patients described feelings of frustration (35\%), anger (24\%) and low self-esteem (22\%), as well as feeling misunderstood (26\%) and worthless (22\%) (fig. 2). Around one-quarter of patients reported gaining little pleasure from the activities they used to enjoy and some felt fearful or frightened. Two-thirds of carers reported feeling fearful of what the future holds for their relative and $22 \%$ often or very often felt stressed as a result of the care they needed to provide (fig. 2). Furthermore, one-fifth of carers were uncertain about what to do for their patient (19\%), or were concerned about either not doing enough for their patient $(21 \%)$ or not doing well enough as a carer $(20 \%)$. The qualitative interviews revealed that onethird of carers and over half of patients (55\%) felt socially isolated due to the lack of understanding of PAH among family and friends, as well as the general public. Almost one-third of carers also experienced no understanding of their situation when they informed other people about being a carer (29\%). Despite these difficulties, carers tend to prioritise the patients' emotions above their own, and $61 \%$ of carers found that this had a very significant impact on their daily life. On average, in a normal month, carers spent as much time on activities for their patient as on activities for themselves. Although one-third of carers found no fulfilment in being a carer, they also reported many positive aspects, with $92 \%$ of carers feeling that they are contributing to the patient's QoL and nearly two-thirds feeling that caring had brought them closer as a couple or family.

\section{Patients and carers need more information on the wider impact of PAH}

It became clear during the qualitative interviews that patients and carers require more information on the wider impact of PAH. Feedback from the patients suggests there is a lack of information on the emotional impact of the disease and, although this information is often given verbally at the time of diagnosis, physical aspects of the disease were frequently the main focus. This is reflected by over one-third (36\%) of patients reporting that they require more information about depression and other emotional consequences of PAH (fig. 3). In addition, patients wish to receive more information on treatment options (67\%), the disease itself (61\%) and administrative consequences of PAH (55\%) (fig. 3). Patients look to patient organisations to support their information (66\%) and emotional (30\%) needs, but many are not directed to this important source of support by their HCPs. Similar information is requested by carers of PAH patients and they actively seek information from a variety of sources. Carers are keen to receive regular updates on their relative or the patient they care for and express an interest in receiving information on specialists involved in PAH care, social and financial support, and emotional consequences of living with PAH. Moreover, both patients and carers indicated that information should be provided in a staggered manner.

\section{Implications for the future management of PAH}

The results from this international survey illustrate the profound impact PAH has on all aspects of life, both for patients and carers. A replica of this survey taken by PAH patients and carers in the USA confirmed 


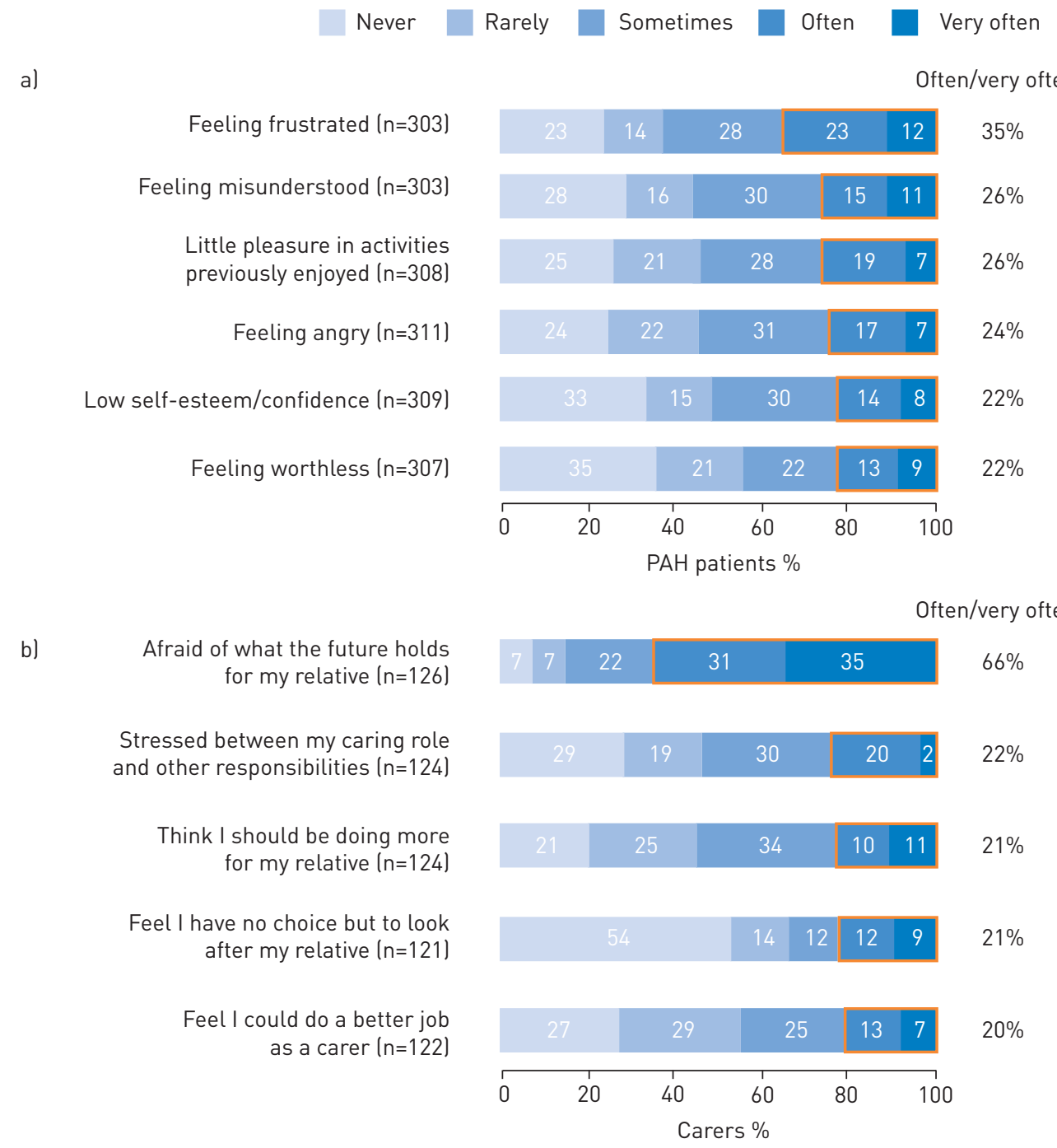

FIGURE 2 Frequency of emotions experienced by a) pulmonary arterial hypertension (PAH) patients and b) their carers.

these observations, with over $60 \%$ of both patients and caregivers reporting a very significant impact of $\mathrm{PAH}$ on their lives and good agreement on the key aspects of life that were most affected by the disease [19].

Qualitative studies on patient and partner/carer experiences of living with advanced colorectal cancer, rheumatoid arthritis and asthma have identified similar issues to those discussed here. For patients, these included the need for honest communication and knowledge about their disease and prognosis, making changes to their work and consequent financial difficulties [20], and feeling alone because the disease is not visible to others and almost impossible to understand for someone not suffering from it [21]. Partners of patients reported making substantial changes to most aspects of their daily life, including work in some cases [22], an increased burden of responsibility and daily chores [20], and a negative impact on intimacy $[20,22]$. They also described feelings of fear or isolation due to friends or acquaintances disappearing [20], and expressed their desire to receive more information on available support [22].

In accordance with the findings from this survey, high rates of anxiety/depression have been reported in patients with advanced nonsmall cell lung cancer [23], rheumatoid arthritis [24, 25] and severe asthma [26]. Moreover, a negative impact of chronic disease on patients' mental function [24, 27], role, emotional or social functioning $[24,28,29]$, and both the intensity and quality of sexual relationships [30] has been described. With regards to family caregivers and other family members, high levels of stress, which are associated with reduced caregiver QoL [31], and decreased mental health-related QoL [32] have been documented. Of note, in diseases such as advanced cancers and asthma, attempts to lower stress and improve QoL and emotional function by structured multidisciplinary interventions have shown promising results both for patients [33-36] and carers [34]. 


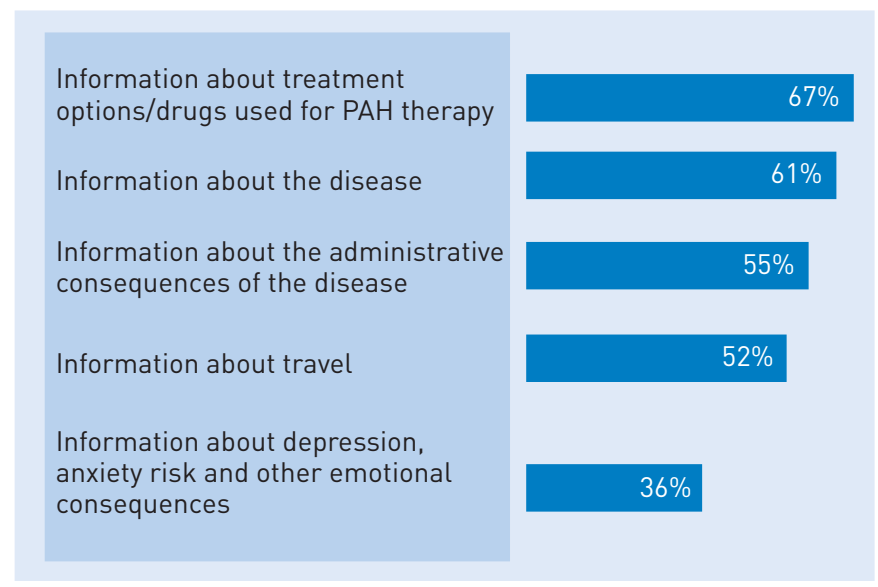

FIGURE 3 The main information needs for 317 patients with pulmonary arterial hypertension (PAH).

Considering these data and the findings of this survey, it is the opinion of the Pulmonary Hypertension Associations of Europe, the UK and the USA that a number of initiatives are required to ensure there is a comprehensive standard of care in the management of PAH. Such initiatives are essential to improve the physical, practical, emotional and social well-being of $\mathrm{PAH}$ patients and carers, and to address their information needs. Optimal management of PAH requires a coordinated approach from a multidisciplinary team of HCPs and other specialists, including physicians, nurses, social workers, psychologists and patient organisations. This research has highlighted the significant impact that caring for someone with $\mathrm{PAH}$ has on the carer themselves. Therefore, it is imperative that future initiatives ensure that the carer is also given due consideration and that their needs are addressed as part of the patient's multidisciplinary package of care. It is also important to emphasise that the disease burden of PAH (practical, physical, emotional and social) increases significantly with a decline in functional class. It is critical that awareness of the non-physical impact of PAH is raised amongst HCPs, including the effect it has on patients and carers.

Therefore, future PAH guidelines should strongly emphasise the need to provide more information on all aspects of PAH to both patients and carers. Moreover, it should be recommended that patients and their carers are pro-actively referred to sources of additional support depending on their individual needs, e.g. adequate psychological support, occupational counselling or administrative advice.

Patient organisations have emerged as a key resource for providing information and support to both patients and carers; therefore, it is important that relationships are strengthened between HCPs and patient groups. Patients under specialist care, and their carers, should be directed to patient groups early after diagnosis in order to gain further support and information and access to the PAH community. Stronger links between patient groups and HCPs can support the development and timely provision of appropriate information on topics beyond the physical impact of the disease. Results from the survey revealed that the information that patients would like to receive at diagnosis was related to the disease itself and its treatment, and that this differed from the type of information they would like to receive at later stages (1, 3 and 6 months, and $\geqslant 12$ months post-diagnosis). This may mean that information on physical and practical, emotional, social and financial aspects may be more appropriate at follow-up visits, rather than at diagnosis when patients may be overwhelmed with information. HCPs should receive training on how best to tailor and communicate information to the patient.

The results of this survey have major implications for the scope of care provided to patients with PAH. They demonstrate that PAH guidelines need to be updated to reflect the multidimensional care necessary to address the physical and practical, emotional, social and information needs of both patients and carers, alongside their clinical needs.

\section{Conclusion}

The International PAH Patient and Carer Survey provided invaluable insight into the wider impact of PAH. The immense burden imposed by PAH exacts an emotional toll on both patients and carers, resulting in a wide range of negative emotions. Many patients and carers feel concerned about what the future may hold, highlighting the clear need to address the psychosocial needs of both patients and their carers. Living with $\mathrm{PAH}$ is difficult and its impact on QoL is far-reaching. It is imperative that patients and carers are aware that the burden of PAH goes beyond the clinical symptoms and feel confident in requesting support from the appropriate healthcare/patient groups to address the physical and practical, social, emotional and 
financial aspects of the disease. This study is an important step towards improving all aspects of life for PAH patients and their carers and providing care that meets these needs.

\section{Acknowledgements}

We would like to thank the PAH patients and carers who took part in the survey and the patient associations for their valuable input into the planning and recruitment of the research.

This survey has been developed under the auspices of an Actelion Pharmaceuticals Ltd (Allschwil, Switzerland) sponsored steering committee comprising of PAH experts. Medical writing support was provided by S. Mariotti Nesurini (nspm UK Ltd, Cheadle, UK), funded by Actelion Pharmaceuticals Ltd.

\section{References}

1 Galiè N, Hoeper MM, Humbert M, et al. Guidelines for the diagnosis and treatment of pulmonary hypertension: the Task Force for the Diagnosis and Treatment of Pulmonary Hypertension of the European Society of Cardiology (ESC) and the European Respiratory Society (ERS), endorsed by the International Society of Heart and Lung Transplantation (ISHLT). Eur Heart J 2009; 30: 2493-2537.

2 Rich S, Dantzker DR, Ayres SM, et al. Primary pulmonary hypertension. A national prospective study. Ann Intern Med 1987; 107: 216-223.

3 Vonk-Noordegraaf A, Galiè N. The role of the right ventricle in pulmonary arterial hypertension. Eur Respir Rev 2011; 20: 243-253.

4 Humbert M, Simonneau G, Rubin LJ. A decade of achievement in pulmonary hypertension. Eur Respir Rev 2011; 20: $215-227$.

5 Humbert M, Souza R, Galie N, et al. Pulmonary arterial hypertension: bridging the present to the future. Eur Respir Rev 2012; 21: 267-270.

6 Vachiéry JL, Gaine S. Challenges in the diagnosis and treatment of pulmonary arterial hypertension. Eur Respir Rev 2012; 21: 313-320.

7 Gihl AF. Health-related quality of life in pulmonary arterial hypertension. Adv Pulm Hypertens 2013; 8: 215-222.

8 Rubenfire M, Lippo G, Bodini BD, et al. Evaluating health-related quality of life, work ability, and disability in pulmonary arterial hypertension: an unmet need. Chest 2009; 136: 597-603.

9 Lowe B, Grafe K, Ufer C, et al. Anxiety and depression in patients with pulmonary hypertension. Psychosom Med 2004; 66: 831-836.

10 Shafazand S, Goldstein MK, Doyle RL, et al. Health-related quality of life in patients with pulmonary arterial hypertension. Chest 2004; 126: 1452-1459.

11 White J, Hopkins RO, Glissmeyer EW, et al. Cognitive, emotional, and quality of life outcomes in patients with pulmonary arterial hypertension. Respir Res 2006; 7: 55.

12 Badesch DB, Raskob GE, Elliott CG, et al. Pulmonary arterial hypertension: baseline characteristics from the REVEAL Registry. Chest 2010; 137: 376-387.

13 Looper KJ, Pierre A, Dunkley DM, et al. Depressive symptoms in relation to physical functioning in pulmonary hypertension. J Psychosom Res 2009; 66: 221-225.

14 McCollister DH, Beutz M, McLaughlin V, et al. Depressive symptoms in pulmonary arterial hypertension: prevalence and association with functional status. Psychosomatics 2010; 51: 339.e8.

15 Armstrong I, Harries C, Yorke J. The Impahct Survey: living with pulmonary arterial hypertension. Am J Respir Crit Care Med 2011; 183: A4130.

16 Flattery MP, Pinson JM, Savage L, et al. Living with pulmonary artery hypertension: patients' experiences. Heart Lung 2005; 34: 99-107.

17 McDonough A, Matura LA, Carroll DL. Symptom experience of pulmonary arterial hypertension patients. Clin Nurs Res 2011; 20: 120-134.

18 McKenna SP, Doughty N, Meads DM, et al. The Cambridge Pulmonary Hypertension Outcome Review (CAMPHOR): a measure of health-related quality of life and quality of life for patients with pulmonary hypertension. Qual Life Res 2006; 15: 103-115.

19 Pulmonary Arterial Hypertension Association. The impact of pulmonary arterial hypertension (PAH) on the lives of patients and caregivers: results from a US study. www.phaonlineuniv.org/files/PHA\%20Pub_Final\%203\% 2028\%202013.pdf Date last updated: March 28, 2013. Date last accessed: July 19, 2013.

20 Sjovall K, Gunnars B, Olsson H, et al. Experiences of living with advanced colorectal cancer from two perspectives inside and outside. Eur J Oncol Nurs 2011; 15: 390-397.

21 Trollvik A, Nordbach R, Silen C, et al. Children's experiences of living with asthma: fear of exacerbations and being ostracized. J Pediatr Nurs 2011; 26: 295-303.

22 Matheson L, Harcourt D, Hewlett S. "Your whole life, your whole world, it changes": partners' experiences of living with rheumatoid arthritis. Musculoskeletal Care 2010; 8: 46-54.

23 Chouaid C, Agulnik J, Goker E, et al. Health-related quality of life and utility in patients with advanced non-smallcell lung cancer: a prospective cross-sectional patient survey in a real-world setting. $J$ Thorac Oncol 2013; 8: 997-1003.

24 Hodkinson B, Musenge E, Ally M, et al. Functional disability and health-related quality of life in South Africans with early rheumatoid arthritis. Scand J Rheumatol 2012; 41: 366-374.

25 Hyphantis T, Kotsis K, Tsifetaki N, et al. The relationship between depressive symptoms, illness perceptions and quality of life in ankylosing spondylitis in comparison to rheumatoid arthritis. Clin Rheumatol 2013; 32: 635-644.

26 Yonas MA, Marsland AL, Emeremni CA, et al. Depressive symptomatology, quality of life and disease control among individuals with well-characterized severe asthma. J Asthma 2013; 50: 884-890.

27 Sullivan PW, Smith KL, Ghushchyan VH, et al. Asthma in USA: its impact on health-related quality of life. J Asthma 2013; 50: 891-899.

28 Larsson M, Ljung L, Johansson BB. Health-related quality of life in advanced non-small cell lung cancer: correlates and comparisons to normative data. Eur J Cancer Care (Engl) 2012; 21: 642-649. 
Schaake W, de GM, Krijnen WP, et al. Quality of life among prostate cancer patients: a prospective longitudinal population-based study. Radiother Oncol 2013; 108: 299-305.

30 Rouanne M, Massard C, Hollebecque A, et al. Evaluation of sexuality, health-related quality-of-life and depression in advanced cancer patients: a prospective study in a phase I clinical trial unit of predominantly targeted anticancer drugs. Eur J Cancer 2013; 49: 431-438.

31 Bellin $\mathrm{MH}, \mathrm{Kub}$ J, Frick KD, et al. Stress and quality of life in caregivers of inner-city minority children with poorly controlled asthma. J Pediatr Health Care 2013; 27: 127-134.

32 Sjolander C, Rolander B, Jarhult J, et al. Health-related quality of life in family members of patients with an advanced cancer diagnosis: a one-year prospective study. Health Qual Life Outcomes 2012; 10: 89.

33 Chock MM, Lapid MI, Atherton PJ, et al. Impact of a structured multidisciplinary intervention on quality of life of older adults with advanced cancer. Int Psychogeriatr 2013; 4: 1-10.

34 Lengacher CA, Kip KE, Barta M, et al. A pilot study evaluating the effect of mindfulness-based stress reduction on psychological status, physical status, salivary cortisol, and interleukin-6 among advanced-stage cancer patients and their caregivers. J Holist Nurs 2012; 30: 170-185.

35 Pbert L, Madison JM, Druker S, et al. Effect of mindfulness training on asthma quality of life and lung function: a randomised controlled trial. Thorax 2012; 67: 769-776.

36 Piderman KM, Johnson ME, Frost MH, et al. Spiritual quality of life in advanced cancer patients receiving radiation therapy. Psychooncology 2013 [in press DOI: 10.1002/pon.3390]. 